\title{
Numerical Analysis of a Rotating Detonation Engine in the Relative Reference Frame
}

\author{
Daniel E. Paxson ${ }^{1}$ \\ NASA Glenn Research Center, Cleveland, Ohio, 44130
}

\begin{abstract}
A two-dimensional, computational fluid dynamic (CFD) simulation of a semi-idealized rotating detonation engine (RDE) is described. The simulation operates in the detonation frame of reference and utilizes a relatively coarse grid such that only the essential primary flow field structure is captured. This construction yields rapidly converging, steady solutions. Results from the simulation are compared to those from a more complex and refined code, and found to be in reasonable agreement. The performance impacts of several RDE design parameters are then examined. Finally, for a particular RDE configuration, it is found that direct performance comparison can be made with a straight-tube pulse detonation engine (PDE). Results show that they are essentially equivalent.
\end{abstract}

\section{Nomenclature}

$a_{*} \quad=$ non-dimensional speed of sound

$a^{*} \quad=$ dimensional reference speed of sound

$A F R \quad=$ air to fuel ratio (by mass)

$\varepsilon \quad=$ ratio of inlet (or exit) slit height to annulus height

$\underline{F}=$ non-dimensional conserved circumferential flux vector

$g_{c} \quad=$ Newton constant

$\underline{G} \quad=$ non-dimensional conserved axial flux vector

$\gamma \quad=$ ratio of specific heats

$h_{f} \quad=$ fuel heating value

$I \quad=$ Impulse

$K_{0} \quad=$ non-dimensional reaction rate constant

$l \quad=$ circumferential length

$L=$ tube length

$M_{y} \quad=\quad$ Axial Mach number

$p_{*} \quad=$ non-dimensional pressure

$p^{*} \quad=$ dimensional reference pressure

$q_{0}=$ non-dimensional heat addition parameter

$R_{g} \quad=$ real gas constant

$\rho \quad=$ non-dimensional density

$\rho^{*} \quad=$ dimensional reference density

$\underline{S} \quad=$ non-dimensional source term vector

$T=$ non-dimensional temperature

$T^{*} \quad=$ dimensional reference temperature

$T_{c 0}=$ non-dimensional reaction temperature

$T_{s p} \quad=$ specific thrust

$t \quad=$ non-dimensional time

$u=$ non-dimensional circumferential velocity

$v \quad=$ non-dimensional axial velocity

$\underline{w}=$ non-dimensional conserved state vector

$x=$ non-dimensional circumferential distance

\footnotetext{
${ }^{1}$ Aerospace Research Engineer, Controls and Dynamics Branch, 21000 Brookpark Road/MS 77-1, AIAA Associate Fellow
} 
$\begin{array}{ll}y & =\text { non-dimensional axial distance } \\ z & =\text { reactant fraction }\end{array}$

$\begin{array}{ll}\text { Subscripts } & \\ e & =\text { exit plane } \\ b & =\text { back (as in back or ambient pressure) } \\ \text { det } & \text { referring to the detonation } \\ t r & =\text { total (pressure or temperature) in reservoir }\end{array}$

\section{Introduction}

$\mathrm{T}$

he rotating detonation engine (RDE) represents an intriguing approach to achieving pressure gain combustion (PGC) for propulsion and power systems. The RDE essentially consists of an annulus with one end open (or having a nozzle) and the other end having a valve system (possibly using non-mechanical, fluidic actuation). Fuel and oxidizer enter axially through the valved end. The detonation travels circumferentially. Combustion products exit, predominantly axially, through the open end. The process is illustrated in Fig. 1, which shows computed contours of normalized temperature throughout the 'unwrapped' annulus at a moment in time during the cycle. Also shown in the figure are crude, but roughly scaled inlet and exit axial velocity vectors. The majority of the fluid entering the device is passed over or 'processed' by the rotating detonation wave which substantially raises the pressure and temperature. As the fluid continues to move predominantly axially, it is expanded and accelerated. When it leaves the device, the fluid pressure and momentum are elevated relative to the inlet. As such, it produces thrust and/or average total pressure gain.

The potential advantages of this approach compared to conventional pulsed detonation engines (PDE's) include the elimination of initiation and deflagration to detonation transition (DDT) devices, and an exceptionally high cycle rate governed by the transit time of the detonation around the circumference.

Although these devices have been demonstrated in the laboratory ${ }^{1,2}$, they are difficult to instrument. Additionally, since nearly all laboratory devices do not have mechanical valves, they cannot be safely operated with premixed fuel and air. As such there is significant uncertainty about local stoichiometry and therefore detonation properties. The net result of these difficulties is that, while RDE's are operational, their fundamental physical principles are not well understood. Not surprisingly, neither are methodologies to optimize performance.

Computational fluid dynamic (CFD) studies have been performed, ${ }^{3,4}$; however, they are relatively few in

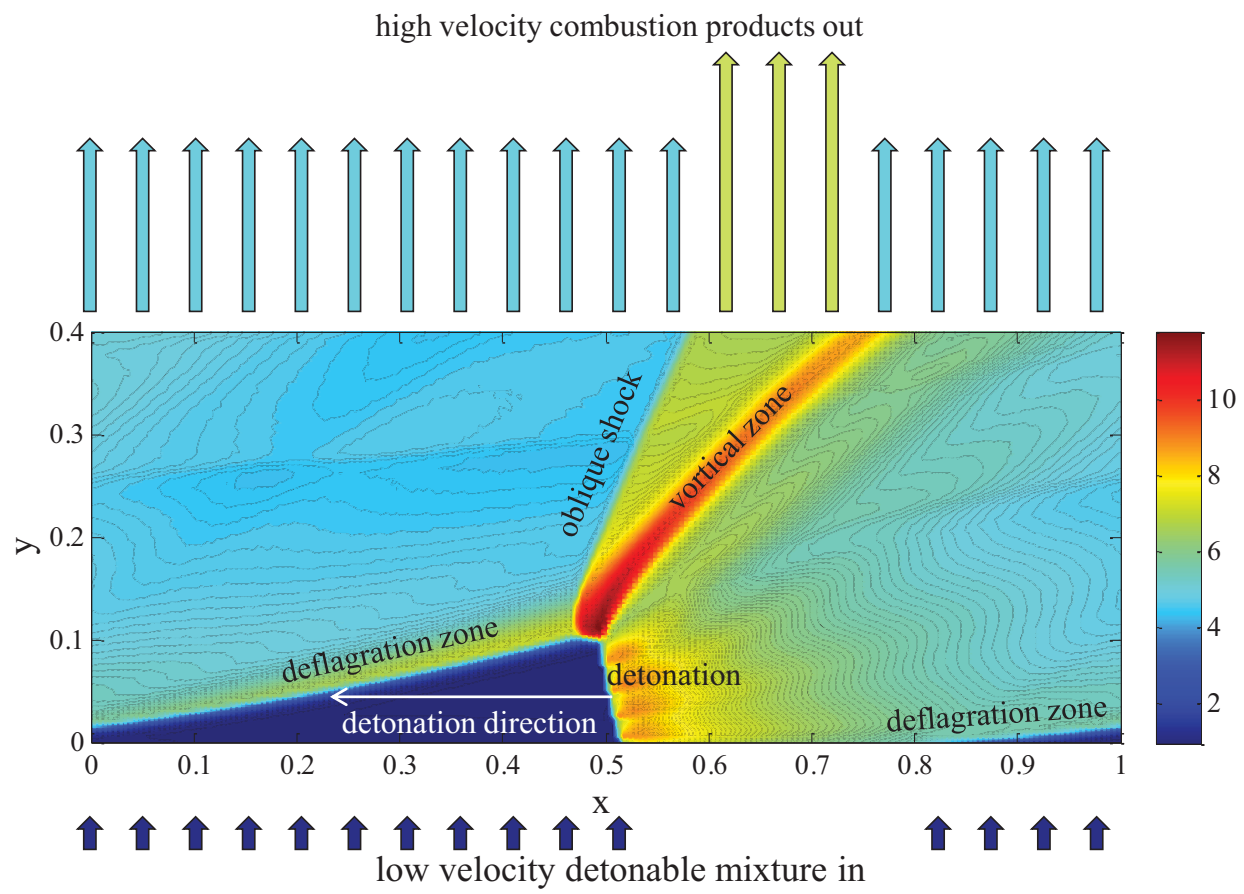

Figure 1. Computed contours of non-dimensional temperature throughout the annulus of an 'unwrapped' RDE, at a moment in time during the cycle. 
comparison to those for PDE's. As such there is a need for further and varied computational studies in order to compare against each other, and with the growing body of experiments. Furthermore, many of the existing computational models are constructed with the goal of capturing tremendous detail in the flow field. This implies fine grid spacing, and complex reaction models that, while yielding valuable data, require vast computational resources and time in order to reach convergence. For the sort of parametric evaluations needed to understand fundamental operational physics, a simpler, less refined modeling approach is beneficial.

This paper presents the preliminary results of just such an approach. A CFD-based RDE model is described whereby the reference frame of the rotating detonation is adopted. As such, converged solutions are obtained which are steady in time. This is insured by utilizing a relatively coarse grid and a simple finite rate reaction model which respectively eliminate the otherwise unstable shear flow, and the cellular detonation structure associated with more detailed analyses. Comparison with the more detailed CFD models will show that these simplifications preserve performance prediction capability, and by extension, the capture of essential RDE physics. The use of a coarse grid also yields converged solutions rapidly while utilizing modest computational resources. This feature makes the model useful for parametric studies related to performance.

The flexibility of the model, and its methodological similarity to a quasi-one-dimensional CFD code for investigating conventional pulse detonation engines $(\mathrm{PDE})^{8}$, allow for a direct comparison of semi-idealized RDE and PDE performance. The conditions under which this comparison is made, and the results will be described. The results will show that under these conditions, the performance of RDE's and PDE's is nearly the same.

A simplified configuration of the model will also be described which is used to investigate explanations for the reduced detonation speeds (compared to one-dimensional theory) observed in experiments. It will be shown that the lack of axial confinement associated with RDE's is one possibility.

\section{Model Description}

The model is developed with the assumption that the working fluid is a calorically perfect gas (CPG) of fixed composition. This is a significant simplification which is far from reality, particularly in the vicinity of rotating detonation itself. However, it has been shown that with judiciously chosen average gas properties, ${ }^{8}$ remarkably good agreement with experiments can be obtained. More importantly however, the simplification does not fundamentally impact performance trends or fundamental physics, the capture of which are the goals of the present effort. In addition, the CPG assumption greatly simplifies the mathematics associated with code development.

\section{A. Governing Equations}

On the further assumptions that working fluid is inviscid and adiabatic, and that the RDE annulus radius of curvature is much greater than its height, the governing equations of motion may be written in non-dimensional form as follows.

$$
\frac{\partial \underline{w}}{\partial t}+\frac{\partial \underline{F}}{\partial x}+\frac{\partial \underline{G}}{\partial y}=\underline{S}
$$

where

$$
\underline{w}=\left[\begin{array}{c}
\rho \\
\rho u \\
\rho v \\
\frac{p}{\gamma(\gamma-1)}+\frac{\rho\left(u^{2}+v^{2}\right)}{2} \\
\rho z
\end{array}\right]
$$



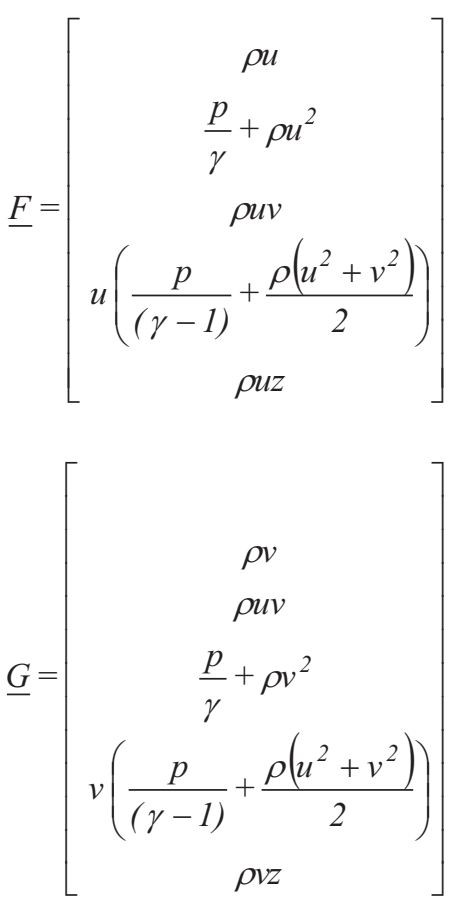

$$
\underline{S}=\left[\begin{array}{c}
0 \\
0 \\
0 \\
\rho z q_{0}\left\{\begin{array}{c}
K_{0} ; T>T_{c 0} \\
0 ; T \leq T_{c 0}
\end{array}\right\} \\
-\rho z\left\{\begin{array}{c}
K_{0} ; T>T_{c 0} \\
0 ; T \leq T_{c 0}
\end{array}\right\}
\end{array}\right]
$$

The non-dimensional equation of state needed to complete the governing equation set is written as follows.

$$
p=\rho T
$$

The non-dimensional pressure, $p$, density, $\rho$, temperature, $T$, and velocities, $u$ and $v$ have been obtained using a reference state $p^{*}, \rho^{*}, T^{*}$, and the corresponding sound speed $a^{*}$. The distances, $x$ and $y$ have been nondimensionalized by the circumference, $l$. The time, $t$ has been non-dimensionalized using the reference wave transit time, $l / a^{*}$. The heat of reaction of the reactant gas mixture, $q_{0}$ is a constant which depends on the assumed fuel heating value, the oxidizer (air in this work), and fuel-to-air ratio corresponding to the reactant fraction, $\mathrm{z}=1.0$.

$$
q_{0}=\frac{h_{f}}{\gamma R_{g} T^{*}(A F R+1)}
$$

For the remainder of the paper, all quantities displayed or discussed are considered non-dimensional unless stated explicitly otherwise.

The finite rate reaction model (Eq. 5) is a very simple one which simply states that the reaction rate is equal to the product of a rate constant, $K_{0}$, and the amount of reactant present. However, the reaction is not allowed to proceed unless the reactants are above a reaction temperature, $T_{c 0}$.

\section{B. Numerical Treatment}

The governing equations are integrated numerically in time using an explicit, second-order, two-step, RungeKutta technique. Spatial flux derivatives are approximated as flux differences, with the fluxes at the discrete cell 
faces evaluated using Roe's approximate Riemann solver ${ }^{5}$. Second-order spatial accuracy (away from discontinuities) is obtained using piecewise linear representation of the primitive variable states within the cells. Oscillatory behavior is avoided by limiting the linear slopes ${ }^{6}$.

\section{Boundary Conditions}

Boundary conditions are imposed by assigning time-dependent values to so-called image (aka "ghost") cells, which are just outside of the interior computing domain. Referring to Fig. 1, the following techniques are used to determine values. At $x=0.0$ and $x=1.0$, symmetry is invoked. This means the rightmost boundary image cells are assigned the value of the leftmost interior cells at each y-location. Similarly the leftmost boundary image cells are assigned the value of the rightmost interior cells. The symmetry assignment insures that the $\mathrm{x}$-dimension of the computational space faithfully represents an annulus (which is continuous and has no boundary).

At the exhaust end, $y=0.4$, constant pressure outflow is used along with characteristic equations to obtain $\rho$, and $v$ for the image cells. If the resulting flow is sonic, or supersonic, then the imposed pressure is disregarded. If it is exactly sonic then the sonic values of $p, \rho$, and $v$ are assigned. If it is supersonic, then $p, \rho$, and $v$ are extrapolated from the interior ${ }^{7}$. The $\mathrm{x}$-velocity component, $u$ is set equal to the last interior cell at each location. This is a crude

form of extrapolation which simply insures that $\frac{\partial u}{\partial y}=0$, thereby allowing slip between exiting flow and that outside the computing domain (about which nothing is known).

At $y=0.0$ (the inflow face), two different boundary conditions are used depending on the interior flow state. This face is presumably fed by a large reservoir at a fixed total pressure, and temperature. If the interior pressure along this face is greater than the reservoir pressure, $p_{t r}$, as might be found just behind the detonation, then a solid wall type boundary conditions are applied with slip in the $x$ direction. This prevents backflow of hot combustion products into the inlet reservoir. If the interior pressure is less than the reservoir pressure, then partially open inflow boundary conditions are applied as described and validated in Ref. 8 . These determine $p, \rho$, and $v$ for the inflow face image cells. This type of boundary condition assumes that flow enters through a slit which is some fraction, $\varepsilon$ of the annulus height. The slit may be imagined as an infinitesimally short converging nozzle. The flow between the reservoir and the nozzle throat is isentropic. For values of $\varepsilon<1$ however, there is a total pressure mixing loss that takes place across a notional mixing plane separating the nozzle throat from the interior computing domain. In the mixing plane, the magnitude of the total pressure loss varies with the flow rate through the slit and the value of $\varepsilon$.

The $\mathrm{x}$-velocity component, $u$, is prescribed during inflow, and it is here that a reference frame change is implemented. Rather than specify $u=0$ (i.e. no swirl) which is the laboratory or fixed frame condition, the negative of the detonation speed, $u_{\text {det }}$ is prescribed instead. As a result of this change to the detonation reference frame, the computational space becomes one where a steady-state solution is possible. This is the computational equivalent to placing an aircraft model in a wind tunnel and moving the air rather than the model.

\section{Solution Procedure}

The detonation speed is not known a priori. The classical calculation associated with the so-called ChapmanJouguet (CJ) point is no longer reliable, as it is based on the assumption of strictly one-dimensional flow (with heat addition ${ }^{9}$. As such, an initial guess is made for $u_{\text {det }}$ and steady cold flow is established in the domain. The value of $q_{0}$ is temporarily set to zero, allowing the potential for reaction without heat release. A steady heat addition rate prescribed at $\mathrm{x}=0.5$, and extending from $y=0$ to $y=0.1$ is then applied. The simulation is restarted (from the established cold flow state), and run until steady state is again reached. This step allows the internal flow to become heated, thereby allowing the reaction to proceed, even though the heat release rate (and location) is fixed. At this point, the fixed heat addition rate is turned off, and $q_{0}$ is restored to the value corresponding to the fuel/oxidizer mixture of interest. The simulation is then restarted and run for the amount of time corresponding to three annular revolutions of the detonation at the assumed detonation speed. The domain is then examined and if it is seen that the detonation front has moved to the right of $\mathrm{x}=0.5$, then it is apparent that the initial guess at $u_{\text {det }}$ is too high. If the detonation front has moved to the left, then the initial guess is too low. Based on this determination, a new guess is made for $u_{d e t}$, and the simulation is re-run for another three detonation revolutions. The process continues until the detonation front remains stationary and the entire domain stops changing. Because the simulation is typically only 200 X 80 numerical cells (several orders of magnitude coarser than previous work ${ }^{3,4}$ ), this can be accomplished in only minutes on a modern laptop computer. When the simulation appears converged, it is then run for the equivalent of 10 additional cycles as a check on whether the detonation is stationary. After this the total mass and energy fluxes at boundaries are checked to insure conservation (i.e. that residuals are acceptably small). 


\section{E. Deflagration and Detonation Zones}

As illustrated in Fig. 1, the primary heat release mechanism in an RDE is a detonation. However, there is also a region where deflagration occurs. In the absence of a so-called buffer layer (pure air with no fuel) premixed charge is introduced adjacent to hot combustion products. If the flow field, and numerical approximation thereof, were truly inviscid, no reaction would occur in this region. In real flows however, there is always diffusion; and the best CFD schemes cannot completely avoid numerical diffusion even when physical diffusion terms are neglected. Diffusion results in combustion products heating the premixed charge, thereby allowing the chemical reaction to proceed.

The particularly simple reaction mechanism used in the present model, together with the coarse grid which generates relatively high numerical diffusion, can lead to unrealistically high flame speeds in the region labeled 'deflagration zone' in Fig. 1. In fact, so much reaction can occur in the deflagration zone that there is no reactant left to detonate. In order to control this, the reaction rate constant, $K_{0}$ is reduced in specified regions of the computational space. The regions can be altered depending on the configuration simulated; however, they are generally defined as shown in Fig. 2

\section{Results}

\section{A. Preliminary Detonation Speed Investigation}

Before proceeding to a full $\mathrm{RDE}$ simulation, the code was used to investigate a simpler configuration. It has been noted that the propagation speed of the detonation in an RDE is slower than in a conventional PDE. The reasons for this are not clear; however, one possibility may be the lack of confinement at the 'top' of the RDE detonation (i.e. $\mathrm{y} \approx 0.1$ in Fig. 1). Immediately behind the detonation front, this provides a sort of

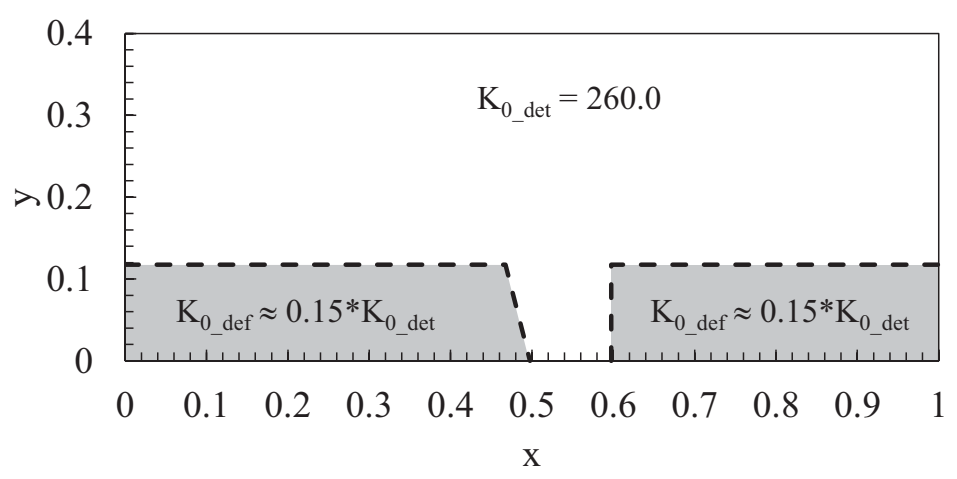

Figure 2. Deflagration and detonation zones in the computing domain.

'leakage path' for energy and momentum that would otherwise propel the detonation forward. In order to test this explanation, the code was run using the boundary conditions and geometry illustrated in Fig. 3.

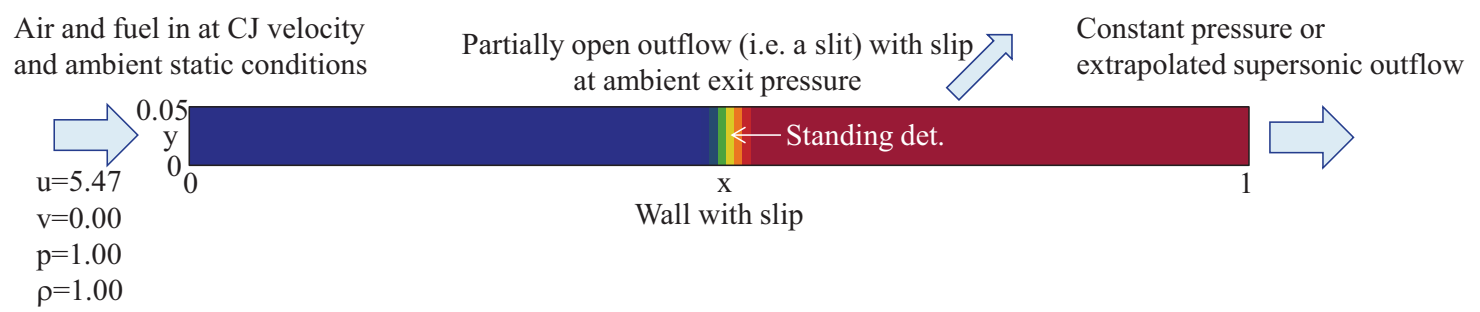

Figure 3. Configuration and boundary conditions for detonation speed investigation. Relevant parameters: $\varepsilon=0.00, \gamma=1.264, T^{*}=520 \mathrm{R}, p^{*}=14.7 \mathrm{psia}, \rho^{*}=0.055 \mathrm{lb}_{\mathrm{m}} / \mathrm{ft}^{3}, \mathrm{a}^{*}=1250 \mathrm{ft} / \mathrm{s}, u_{c j}=5.47, q_{0}=23.4$. The detonable mixture is stoichiometric $h_{2}$ and air. The computational grid is $200 \times 10$ cells.

If the upper surface slit width is set to $\varepsilon=0.0$, then it becomes another wall. No leakage is possible. If the mixture is ignited, and the numerical scheme is working correctly, a purely one dimensional stationary (i.e. steady) detonation should form. In other words, the leftward detonation wave velocity exactly matches the rightward inflow velocity.

The expected outcome of such a test is shown in Fig. 4. The upper portion of the figure shows contours of temperature throughout the domain after 2 non-dimensional time units of simulation. The simulation was initiated (at $\mathrm{t}=0$ ) by setting all numerical cells between $0.0<\mathrm{x}<0.5$ to the predetonative inlet state, and all those between $0.5<\mathrm{x}<1.0$ to the Chapman-Jouguet (CJ) state. The lower portion of Fig. 4 shows the distribution of pressure, temperature, and Mach number at $y=0.025$. Also shown as dashed lines, are the theoretical, post-reactive $\mathrm{CJ}$ values for pressure and temperature. It is clear that a stationary detonation has formed, that the flow is one-dimensional, and that the post reactive $\mathrm{CJ}$ state is properly computed. 


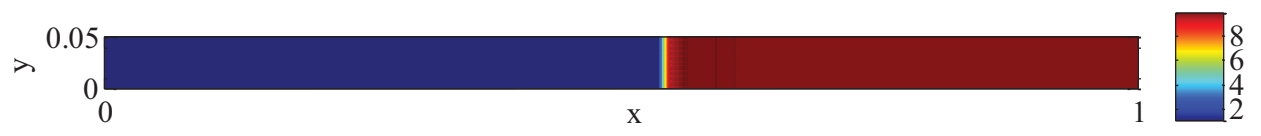

(a)

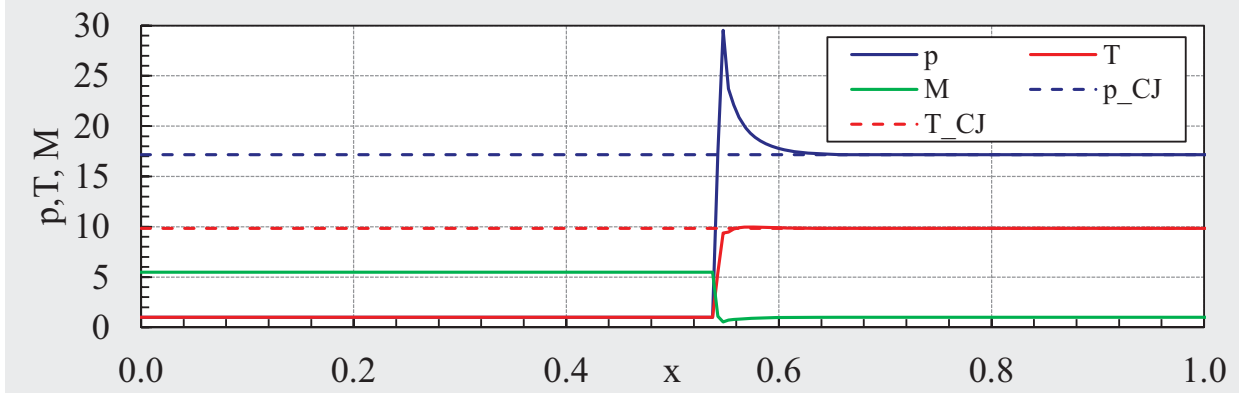

(b)

Figure 4. Computed, steady contours of temperature throughout the Fig. 3 computational test space with $\varepsilon=0.0$ (a); distributions of pressure, temperature and Mach number at $\mathbf{y}=\mathbf{0 . 0 2 5}$ for the same simulation (b).

When the slit width $\varepsilon$, is set to a non-zero value it is observed (though not shown) that the detonation migrates downstream. Given sufficient simulation time, it will ultimately exit the computing domain. This indicates that the detonation speed does indeed decrease when confinement is reduced. In order to recover a stationary wave, the inflow velocity must be reduced. The results of such an exercise are shown in Fig. 5. Here, the same information as Fig. 4 is conveyed; however, the slit width is set to $\varepsilon=0.5$. The inflow velocity required for a stationary detonation is 4.7. This is approximately $14 \%$ below the CJ velocity of 5.47 required for the $\varepsilon=0.0$ case. Figure 5 a also shows two particle streamlines (in white) so that the y-velocity component behind the detonation can be seen. It is interesting to note in the distribution of Fig. 5b that the CJ pressure, temperature and Mach number are never achieved. However, the entropy and total enthalpy rise are exactly the same as Fig. 4. In other words, the reduced pressure rise, and detonation speed do not represent lost availability for the fluid to do work.

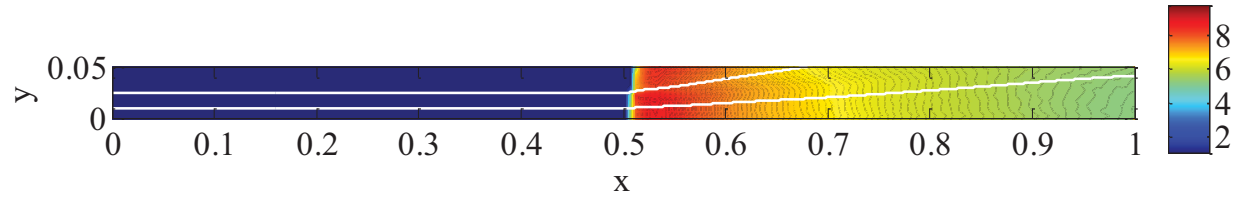

(a)

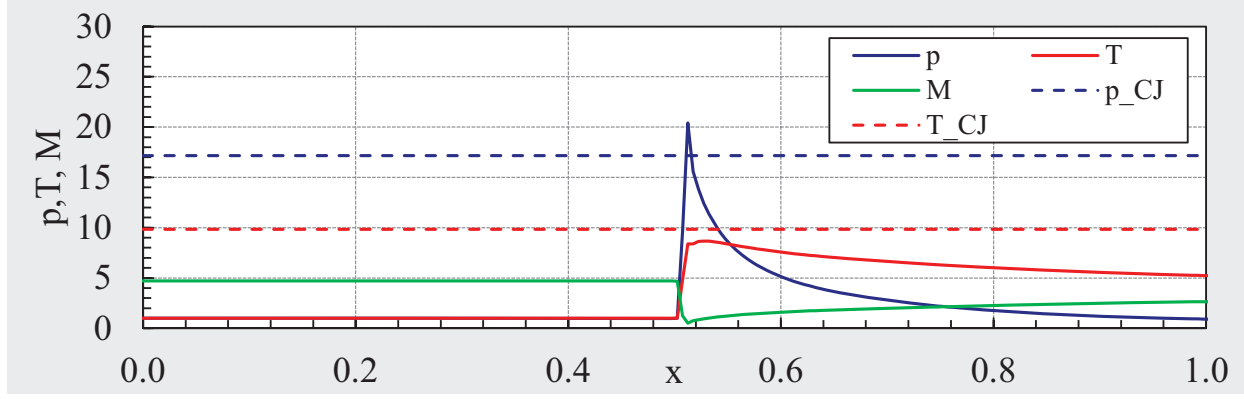

(b)

Figure 5. Computed, steady contours of temperature throughout the Fig. 3 computational test space with $\varepsilon=0.5$ (a); distributions of pressure, temperature and Mach number at $\mathbf{y}=\mathbf{0 . 0 2 5}$ for the same simulation (b).

\section{B. Full RDE Simulation}

The results of a converged simulation of the full RDE domain, under the boundary conditions described earlier, are shown in Fig. 6 in the form of temperature contours. This is similar to Fig. 1. The inlet reservoir pressure and temperature (in the fixed frame) are $p_{t r}=10$ and $T_{t r}=1.038$ respectively. The exit static pressure is $p_{b}=1$. The inlet slit-to-annulus throat height ratio is $\varepsilon=0.20$. The mixture heating value $q_{0}=23.4$ corresponds to stoichiometric 


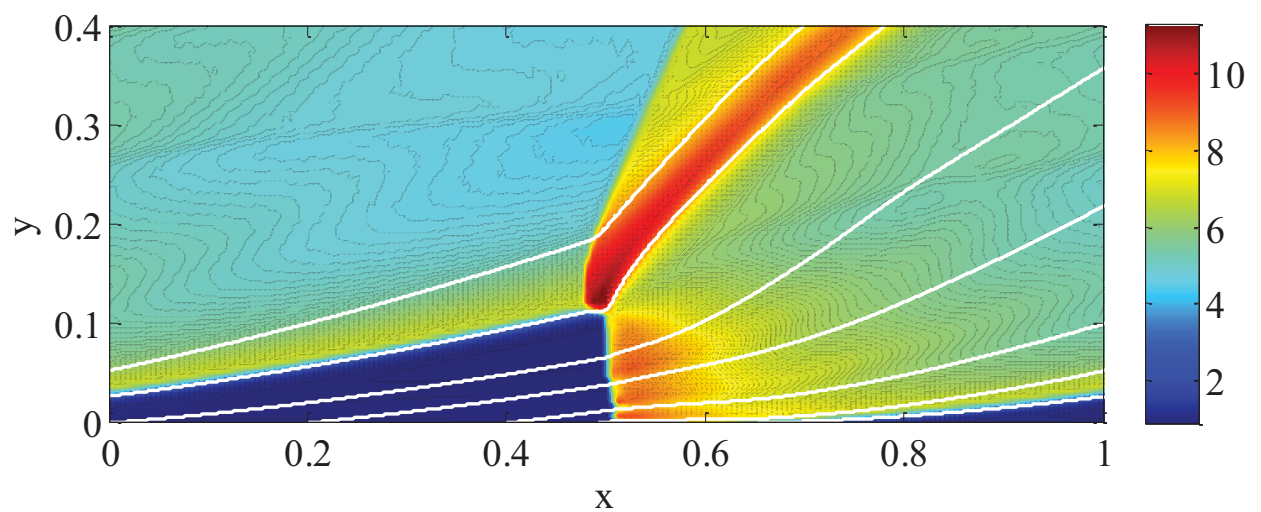

Figure 6. Computed contours of temperature throughout the annulus of an 'unwrapped' RDE, at steady state. Relevant parameters: $p_{t r}=10, T_{t r}=1.038, p_{b}=1$, $\varepsilon=0.20, \gamma=1.264, T^{*}=520 \mathrm{R}, p^{*}=14.7 \mathrm{psia}, \rho^{*}=0.055 \mathrm{lb}_{\mathrm{m}} / \mathrm{ft}^{3}, \mathrm{a}^{*}=1250 \mathrm{ft} / \mathrm{s}, u_{d e t}=4.7$, $q_{0}=\mathbf{2 3 . 4}$.

hydrogen and air. The ratio of specific heats used is $\gamma=1.264$. Dimensional reference conditions are $T^{*}=520 \mathrm{R}$, $p^{*}=14.7 \mathrm{psia}, \rho^{*}=0.055 \mathrm{lb}_{\mathrm{m}} / \mathrm{ft}^{3}, \mathrm{a}^{*}=1250 \mathrm{ft} / \mathrm{s}$. The value of $u_{\text {det }}$ required to achieve a steady solution is $u_{\text {det }}=4.7$. This is $14 \%$ lower than the purely one-dimensional CJ detonation speed for these reference conditions of 5.47 . The computational grid is $200 \times 80$ cells. The features of Fig. 6, including the 'tilt' of the detonation, the height of the detonation, the oblique shock, the deflagration region, and the shear or vortical region appear similar to high fidelity CFD simulations of the same geometry and conditions ${ }^{3,4}$. This does not prove the validity of the simulation; however, it is at least encouraging, particularly given the mere 18 seconds that it takes to compute the flow field for one revolution of the detonation on an ordinary laptop computer.

Also shown in Fig. 6 are select streamlines (in white) which show the paths of particles through the computational domain. It can be seen that most particles enter, pass through the detonation (in this frame of reference), and leave directly through the exit plane. However, some will pass through the oblique shock generated by the detonation wave and will exit at a substantially different state than the other particles. This indicates a nonuniform exhaust flow which will be illustrated later in the paper.

Figure 7 shows the computed gross specific impulse as a function of the ratio of inlet total to exit static pressure. The geometry and working fluid properties are all identical to those used for Fig. 6. Also shown are the computed results from Ref. 3. The Ref. 3 model used a much finer grid resolution (approximately 2 orders of magnitude more cells), a non-calorically perfect gas, and a more sophisticated reaction mechanism than the present model. Furthermore, the partially open inlet was modeled in a different fashion than the present work (a mass source term was used) as were the exit boundary conditions (non-reflecting conditions were imposed). It was also run in the laboratory reference frame. Nevertheless, the performance computed by the two models is quite similar. This provides a measure of confidence in the present approach as a tool for interrogating the flowfield, gaining understanding, and performing rapid parametric analyses.

\section{Discussion}

\section{A. Semi-Idealized Behavior}

The partially open inlet model represents a crude attempt to account for the losses that may be encountered in an actual RDE; however, it has not yet been validated against any experiment. Furthermore, it introduces a loss that is not fundamental. That is to say it can, in theory, be eliminated. As such, it is worth exploring simulations where $\varepsilon=1.0$.

Under this scenario, the performance increases substantially from that shown in Fig. 7. This is evident in Fig. 8 where gross specific impulse at $\varepsilon=1.0$ and 0.20 are compared. This is an expected result as the mixing losses associated with the partially open inlet model can be substantial. However, the performance shown is still considered semi-ideal because there is no exit nozzle modeled and much of the exit flow (in fact all in many cases) is under-expanded. 


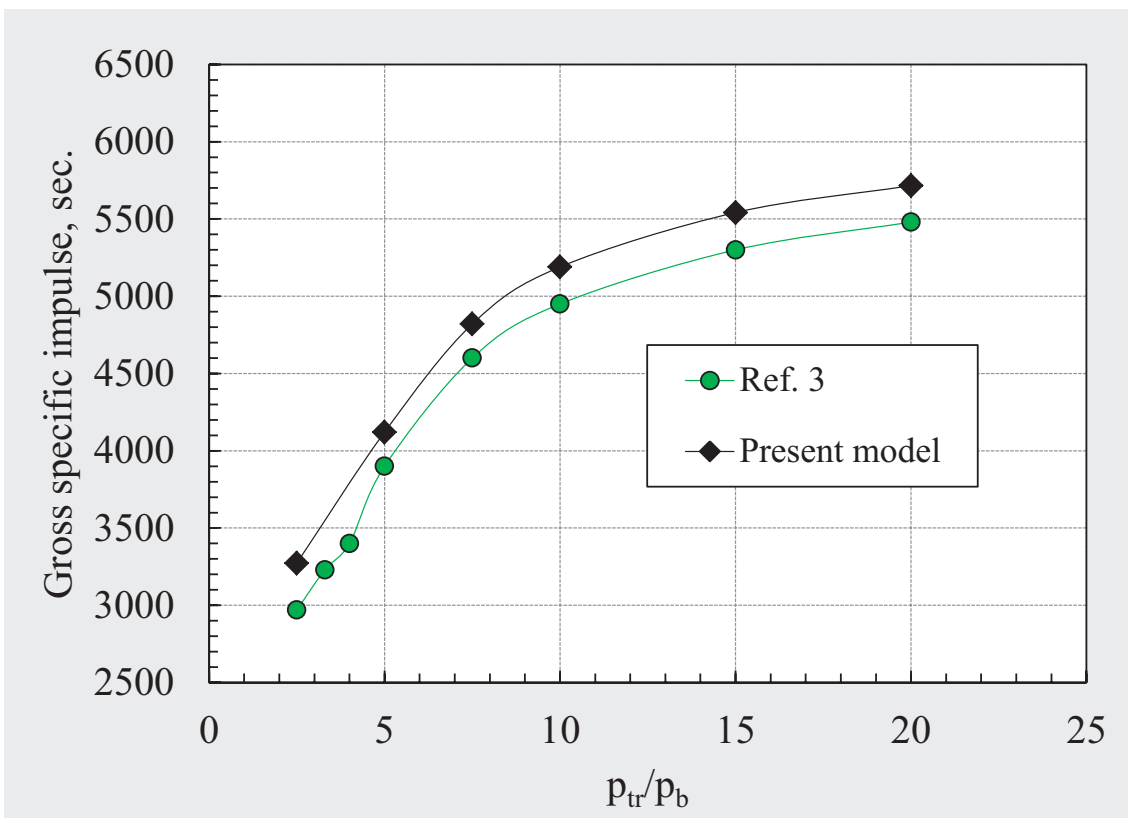

Figure 7. Gross specific impulse as a function of RDE inlet total to exit static pressure ratio for the same geometry and properties used in Fig. 6

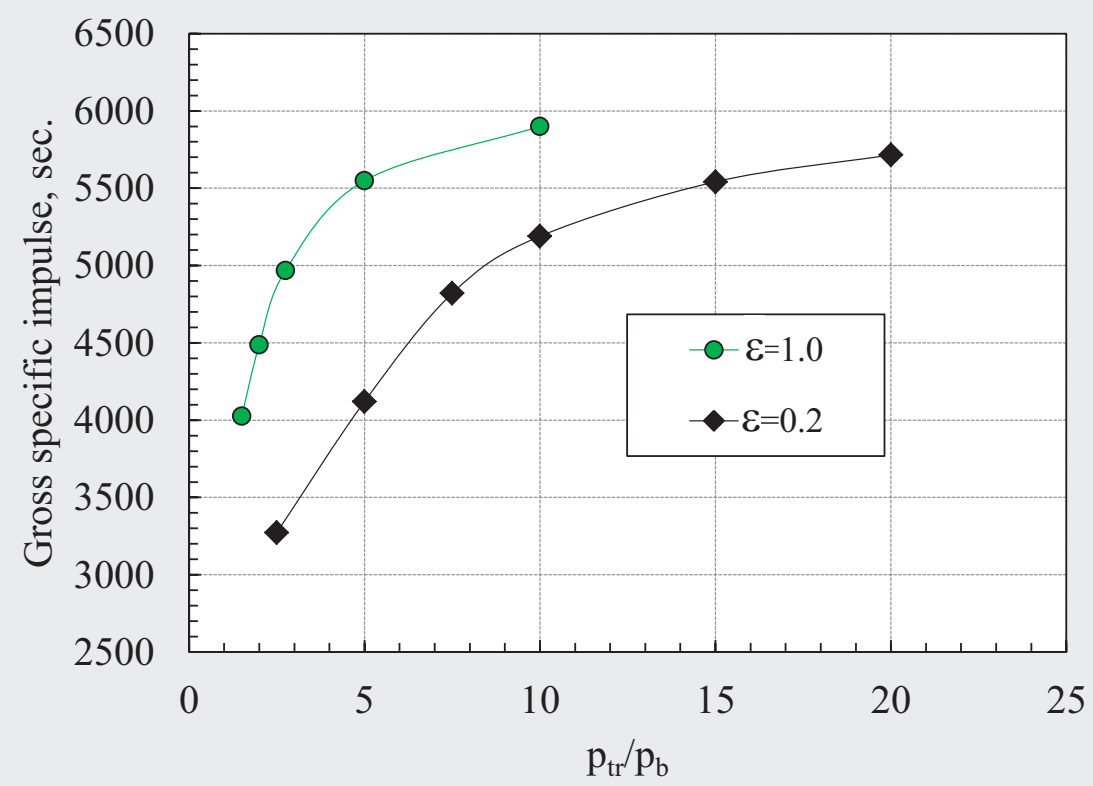

Figure 8. Gross specific impulse as a function of RDE inlet total to exit static pressure ratio for two values of $\varepsilon$, the ratio of inlet throat to annulus height. The geometry and properties are the same as those used in Fig. 6

A detailed examination of the interior indicates that the flow through a portion of the $\varepsilon=1.0 \mathrm{RDE}$ seems to attain a sort of similarity regardless of the inlet pressure. The axial-extent of the detonation becomes fixed. The distribution of axial Mach number and temperature in this region also become fixed. The reason for this appears to be an internal fluidic choking of the flow that occurs downstream (axially) from the detonation. This has the same effect as a choked flow through a physical throat in that Mach number and other distributions become fixed and the exit pressure no longer affects the flow upstream of the throat. An example of the interior axial Mach number distribution is shown in the contour of Fig. 9 for $\varepsilon=1.0$ and $p_{t r}=2.75$. All other parameters are the same as those of Fig. 6. The maximum contour shown (dark red) corresponds to $M_{v}=1.0$. In other words, $M_{y}$ values greater than 1.0 still appear as dark red. It can be seen that there is a somewhat convoluted, but nevertheless nearly continuous (over 


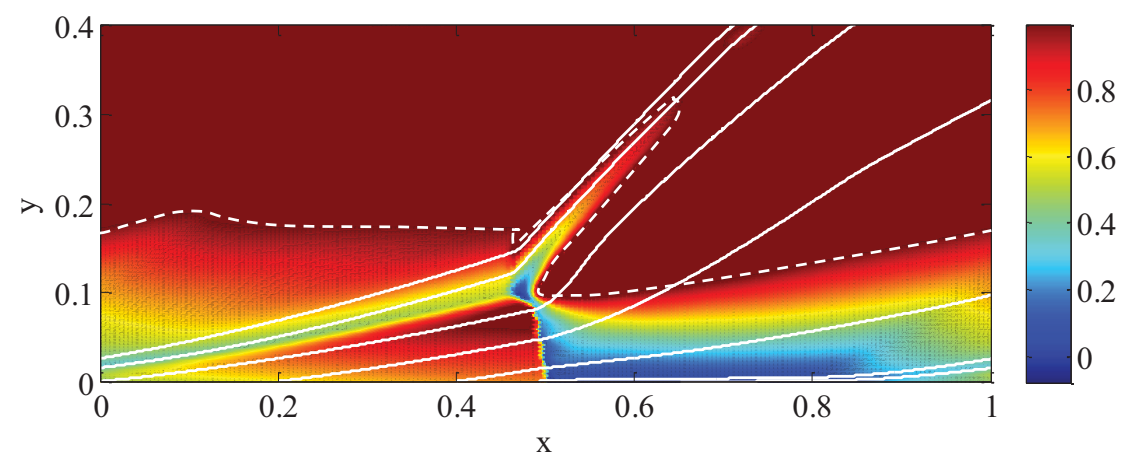

Figure 9. Computed contours of axial Mach number throughout the annulus of an 'unwrapped' RDE, at steady state. The same parameters as Fig. 6 are used except: $p_{t r}=2.75, \varepsilon=1.0$.

all $\mathrm{x}$ locations) contour line representing the 'fluidic throat' whereby the fluid has accelerated from the subsonic values along the inlet plane to sonic values along the contour. This contour is shown as a dashed white line in the figure. The formation of this throat (i.e. choking) helps to explain why the length of an RDE of the configuration shown in this paper has almost no impact on performance. For example, Fig. 10 shows contours of temperature for a semi-ideal RDE under exactly the same conditions as Fig. 9, except the computational domain is only half as long. The computed fuel specific impulse was the same as that of Fig. 9 to within $0.2 \%$. This result may have implications for the design of compact RDE's and for appropriate back-pressurization strategies; however, considerably more investigation is needed.

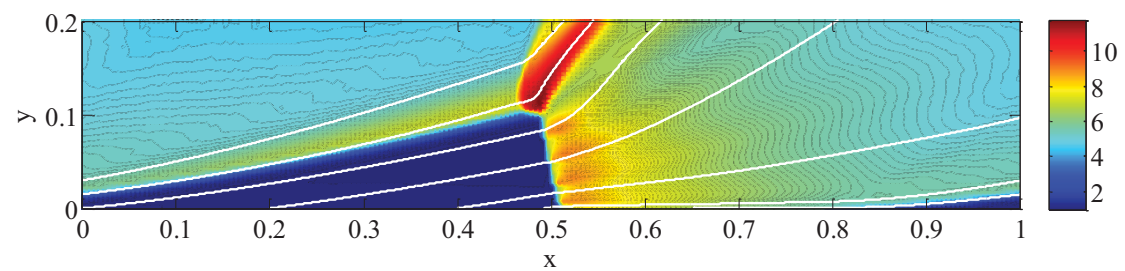

Figure 10. Computed contours of temperature throughout the annulus of an 'unwrapped' RDE, at steady state. The same parameters as Fig. 9 are used.

This fluidic fixing of the flowfield is similar in many ways to the behavior of PDE's when they are equipped with a physical throat at the exhaust end. In order to illustrate this, and to perhaps provide a basis of comparison between RDE's and PDE's, a validated quasi-one-dimensional PDE simulation code ${ }^{9}$ was run using the same idealizations, parameters, and even computational approach as the present RDE code. The PDE simulation contained provisions for a converging exhaust nozzle, and the throat size was adjusted until the time averaged inlet fill Mach number of the PDE matched the space averaged axial inlet fill Mach number of the RDE (approximately 0.54). The converged PDE cycle is shown in Fig. 11 in the form of contours of pressure and temperature. In this figure the vertical axis represents time. The horizontal axis is distance along the PDE tube.

The performance of the RDE and PDE are compared in Fig. 12 which, like Figs. 7 and 8, shows gross specific impulse as a function of inlet total-to-exit static pressure. It can be seen that they are quite similar. The slightly lower RDE performance (approximately 12\% maximum at low pressure ratios) has not been fully explored as of this publication; however, it is likely due to three effects. First, approximately $10 \%$ of the incoming RDE mixture is consumed through less productive deflagration rather than through detonation in the RDE simulation. No deflagration occurs in the PDE simulation. Second, the PDE simulation must include a buffer zone of unfueled inlet air (purge flow) in order to avoid premature ignition of the reactant mixture by the hot gases remaining in the tube during filling. Purge flow often raises fuel specific impulse (while reducing specific thrust) ${ }^{11,12}$. Approximately $3-$ $4 \%$ of the PDE inflow is purge, while there is none in the RDE. Third, the RDE has a unique circumferential velocity element that does not contribute to thrust, but does consume chemical energy. This will be discussed in the next section. 


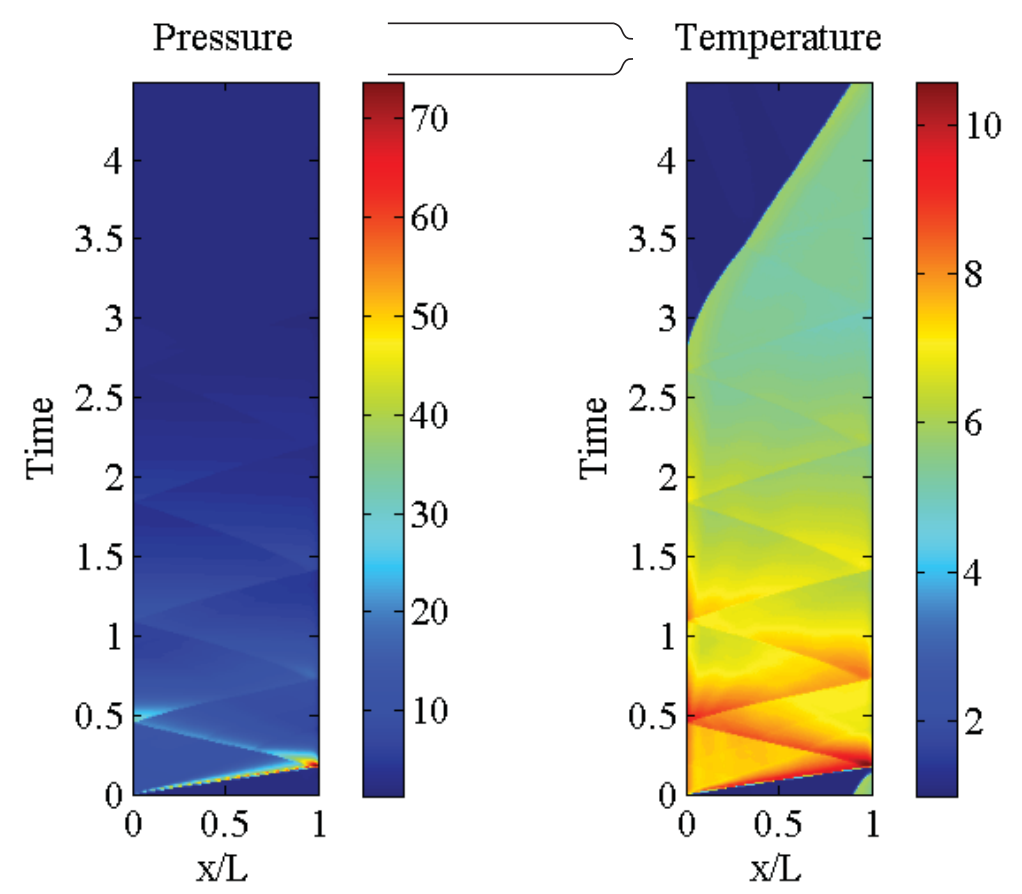

Figure 11. Contours of pressure and temperature over one cycle of a PDE under the same conditions and with the same average inlet Mach number as Fig. 9.

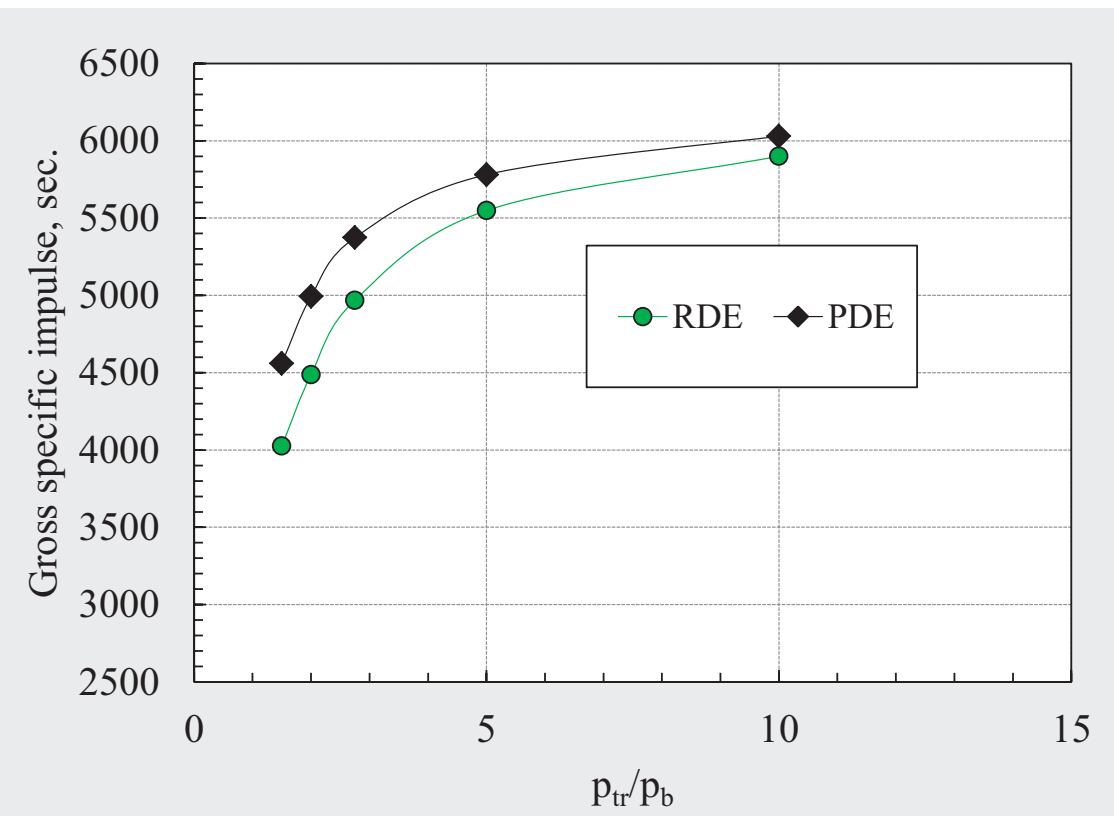

Figure 12. Gross specific impulse as a function of total to static pressure ratio for semi-idealized PDE and RDE at the same average inlet Mach number.

Before doing so however, it is worthwhile to examine the inflow and outflow of the two semi-idealized cycles just described in order see their similarities. This is done in Fig. 13. Figure 13a shows the inflow (axial) Mach number as a function of the fraction of the total cycle mass flow that has entered each device. Figure 13b shows the fraction of the cycle impulse as a function of the fraction of the total cycle mass flow that has exited each device. The origin of both plots coincides with the moment in time when the inlet closes. For reference, the impulse is defined as follows. 


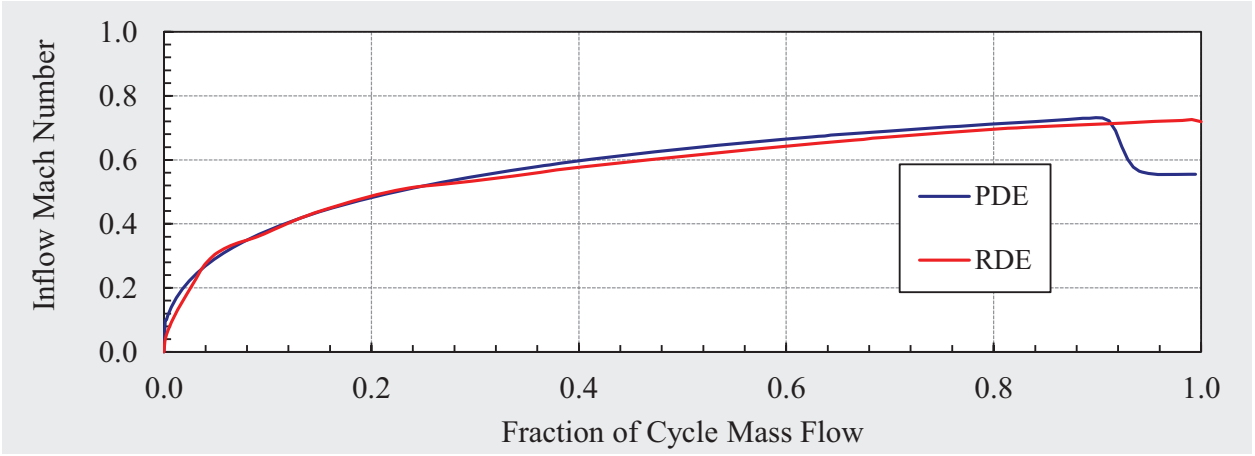

(a)

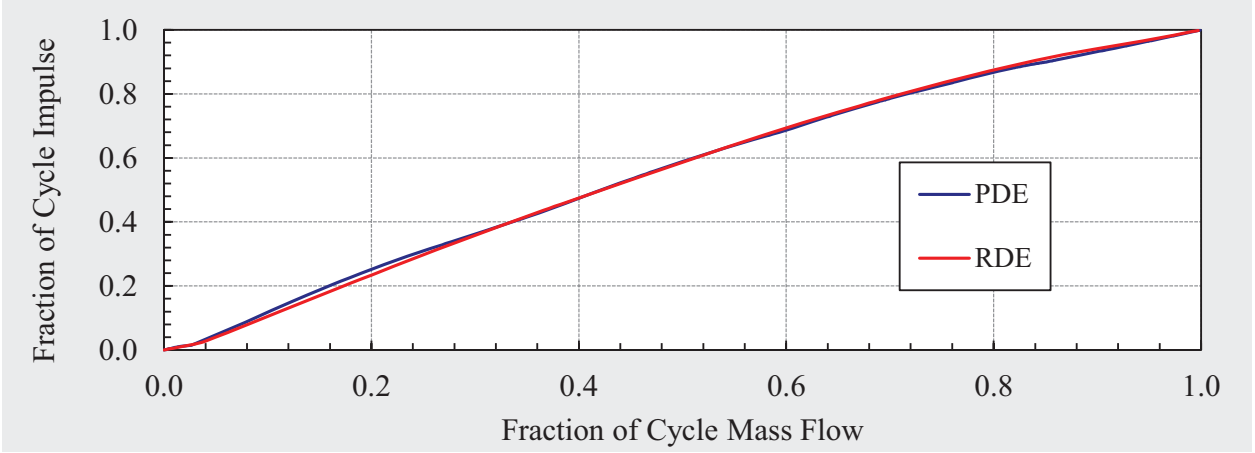

(b)

Figure 13. Inflow axial Mach number as a function of cycle inflow mass flow fraction (a); cycle impulse fraction as a function of cycle exit mass flow fraction (b).

$$
I=\begin{aligned}
& \frac{1}{u_{\text {det }}} \int_{o}^{x}\left(\frac{p_{e}-p_{b}}{\gamma}+\rho_{e} v_{e}^{2}\right) d x-R D E \\
& \int_{o}^{t}\left(\frac{p_{e}-p_{b}}{\gamma}+\rho_{e} u_{e}^{2}\right) d t-P D E
\end{aligned}
$$

Comparing the profiles shown, it is seen that for this semi-idealized cycle, the evolution of thrust and the ingestion of mass are quite similar despite the quite different approaches (RDE vs. PDE) to detonation.

\section{B. Swirl}

1. Exit Swirl

Another contributing factor to the mildly reduced RDE performance compared to the PDE may be due to the swirl (circumferential velocity components) associated with the RDE exit flow. Although the primary flow direction is axial, the transverse direction of the detonation produces swirl. This is shown in Fig. 14 where computed laboratory frame velocity components are plotted as functions of circumferential position in the RDE exit plane for the semi-ideal cycle of Fig. 9. The circumferential component does not contribute to thrust. In fact, it can be shown mathematically that unless swirl is introduced at the inlet to the RDE, no net swirl can exit. In other words, there is no torque present. In terms of the formulation in this paper, this can be stated as follows.

$$
\frac{1}{u_{\text {det }}} \int_{o}^{x}\left(\rho_{e} v_{e}\left\{u_{e}+u_{d e t}\right\}\right) d x=0
$$

When the profile of Fig. 14 is used in Eq. 9, the equality is found to hold to within the numerical accuracy of the code (i.e. the left hand expression of Eq. 9 divided by the impulse from Eq. 8 is less than .009). 


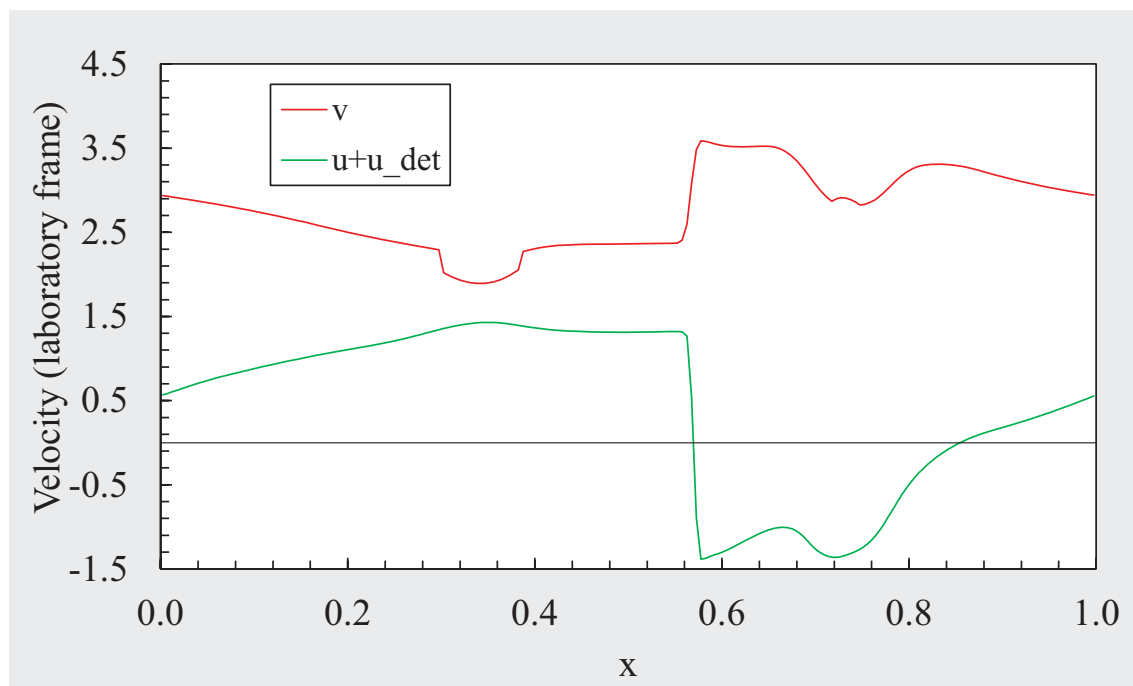

Figure 14. Computed laboratory frame velocity components as functions of circumferential position in the RDE exit plane. Parameters are the same as

Fig. 9.

Although there is no net momentum associated with the locally swirling flow, there is kinetic energy (ultimately converted from the added chemical energy). The computed mass averaged circumferential kinetic energy for the Fig. 14 profile is approximately $2 \%$ of the chemical energy added. This may be written as:

$$
\frac{\int_{o}^{x}\left(\rho_{e} v_{e}\left\{u_{e}+u_{d e t}\right\}^{2}\right) d x}{2 q_{0} \int_{o}^{x}\left(\rho_{e} v_{e}\right) d x}=0.022
$$

\section{Internal Swirl}

The coarse grid of the present simulation causes it to be dissipative. One of the results of this is that lines of slip (which are allowed in inviscid formulations) are manifested as shear layers. The dissipation is numerical; however it may be argued that the effect is physical since real flows are not inviscid. For the RDE simulation, the dissipation of interest occurs at the interface of the flow that has passed through the detonation and is subsequently accelerated (axially and circumferentially), and the flow that is entering through the inlet. This is illustrated in Fig. 15 which shows contours of vorticity for the Fig. 9 conditions. Vorticity is a measure of fluid rotation, but it can also measure shear as it is essentially a combination of spatial velocity gradients. Figure 15 illustrates that the incoming flow retards the post-detonative flow in a dissipative fashion. In other words, it is a form of drag (albeit relatively small).

In closing this section, it is important to emphasize that the results and discussion just presented are preliminary and specific to the configurations used, as well as to the assumptions made. They are intended to demonstrate the

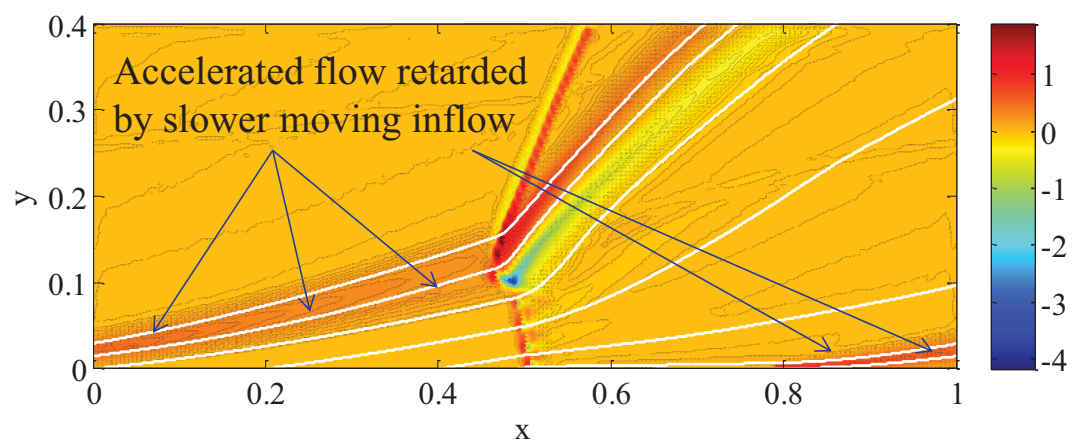

Figure 15. Computed contours of vorticity throughout the annulus of an 'unwrapped' RDE, at steady state. The same parameters as Fig. 9 are used.

13

American Institute of Aeronautics and Astronautics 
utility of the modeling approach described for understanding the physics of RDE's and examining the sensitivity of performance to parametric changes. They are not meant as statements on the practicality of the RDE.

\section{Conclusion}

A relatively simple, two-dimensional, computational fluid dynamic (CFD) based approach to the simulation of rotating detonation engines (RDE's) has been presented. The simplification is achieved by computing in the detonation frame of reference and by utilizing a course computational grid. These result in rapid convergence to steady solutions. Results from the code were shown to compare favorably with those from a more complex and refined code. The capability to assess the impact of RDE configuration changes was demonstrated. So too was the retention of sufficient flowfield detail such that the understanding of fundamental physical processes governing $\mathrm{RDE}$ operation can be ascertained. As part of the latter demonstration, a methodology was described which may allow direct performance comparison of a particular RDE and a pulse detonation engine (PDE). Under this methodology, it was shown that their performance is essentially equivalent. The simplicity of the code means that it can easily be modified to include various well-known loss mechanisms (e.g. heat transfer, wall friction), and configuration modifications such as an exit throat. Such modifications are planned for future versions.

\section{References}

${ }^{1}$ Dyer, R., Naples, A., Kaemming, T., Hoke, J., Schauer, F., "Parametric Testing of a Unique Rotating Detonation Engine Design," AIAA 2012-121, January, 2012.

${ }^{2}$ Naples, Hoke, J., Karnesky, J., Schauer, F., "Flowfield Characterization of a Rotating Detonation Engine," AIAA 20130278, January, 2013.

${ }^{3}$ Schwer, D.A., and Kailasanath, K., "Numerical Investigation of Rotating Detonation Engines," AIAA 2010-6880, July, 2010 .

${ }^{4}$ Schwer, D.A., and Kailasanath, K., "Numerical Study of the Effects of Engine Size on Rotating Detonation Engines," AIAA 2011-581, January, 2011.

${ }^{5}$ Nishikawa, H., Kitamura, K., "Very Simple, Carbuncle-Free, Boundary-Layer-Resolving, Rotated-Hybrid Riemann Solvers," J. of Computational Physics, V. 227, 2008, pp. 2560-2581.

${ }^{6}$ Hirsch, C., Numerical Computation of Internal and External Flows, Vol. 2, Wiley and Sons, NY, 1990, pp. 5493-570.

${ }^{7}$ Paxson, D.E., “A General Numerical Model for Wave Rotor Analysis,”NASA TM 105740, 1992.

${ }^{8}$ Paxson, D.E., “An Improved Numerical Model for Wave Rotor Design and Analysis,” AIAA-93-0482, January, 1993.

Thompson, P. H., Compressible Fluid Dynamics, Department of Mechanical Engineering, Rensselaer Polytechnic Institute, NY, 1988, pp. 349.

${ }^{9}$ Paxson, D.E., Brophy, C.M. and Bruening, G.B., "Performance Evaluation of a Pulse Detonation Combustion Based Propulsion System Using Multiple Methods," JANNAF Journal of Propulsion and Energetics, Vol. 3, No. 1, May, 2010, pp 4454.

${ }^{10}$ Schauer, F., Stutrud, J., Bradley, R., "Detonation Initiation Studies and Performance Results for Pulsed Detonation Engine Applications" AIAA 2001-1129, January 2001.

${ }^{11}$ Paxson, D.E., “A Performance Map for Ideal Air Breathing Pulse Detonation Engines," AIAA-2001-3465 (Also NASA TM 2001-211085), July, 2001. 